\title{
Delivery system for DNAzymes using arginine- modified hydroxyapatite nanoparticles for therapeutic application in a nasopharyngeal carcinoma model
}

\author{
This article was published in the following Dove Press journal: \\ International Journal of Nanomedicine \\ 13 August 2013 \\ Number of times this article has been viewed
}

\section{Yan Chen' \\ Lifang Yang ${ }^{1,2}$ \\ Suping Huang ${ }^{3}$ \\ Zhi $\mathrm{Li}^{\prime}$ \\ Lu Zhang' \\ Jiang $\mathrm{He}^{1}$ \\ Zhijie $\mathrm{Xu}^{2}$ \\ Liyu Liu ${ }^{2}$ \\ $\mathrm{Ya} \mathrm{CaO}^{2}$ \\ Lunquan Sun'}

'Center for Molecular Medicine, Xiangya Hospital, ${ }^{2}$ Cancer Research Institute, ${ }^{3}$ State Key Laboratory of Powder Metallurgy, Central South University, Changsha, People's Republic of China

\section{Video abstract}

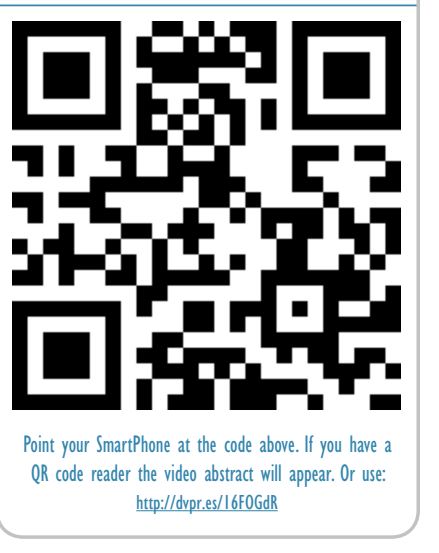

Correspondence: Lifang Yang, Lunquan Sun

Center for Molecular Medicine,

Xiangya Hospital, Central South University, Changsha 410008, People's Republic of China

Tel +8673 I 84327646

Fax +8673184327212

Email yanglifang@csu.edu.cn; lunquansun@csu. edu.cn

\begin{abstract}
DNAzymes are synthetic, single-stranded, catalytic nucleic acids that bind and cleave target mRNA in a sequence-specific manner, and have been explored for genotherapeutics. One bottleneck restricting their application is the lack of an efficient delivery system. As an inorganic nanomaterial with potentially wide application, nanohydroxyapatite particles (nHAP) have attracted increasing attention as new candidates for nonviral vectors. In this study, we developed an nHAP-based delivery system and explored its cellular uptake mechanisms, intracellular localization, and biological effects. Absorption of arginine-modified nanohydroxyapatite particles (Arg-nHAP) and DZ1 (latent membrane protein 1 [LMP1]-targeted) reached nearly $100 \%$ efficiency under in vitro conditions. Using specific inhibitors, cellular uptake of the Arg-nHAP/DZ1 complex was shown to be mediated by the energy-dependent endocytosis pathway. Further, effective intracellular delivery and nuclear localization of the complex was confirmed by confocal microscopy. Biologically, the complex successfully downregulated the expression of LMP1 in nasopharyngeal carcinoma cells. In a mouse tumor xenograft model, the complex was shown to be delivered efficiently to tumor tissue, downregulating expression of LMP1 and suppressing tumor growth. These results suggest that Arg-nHAP may be an efficient vector for nucleic acid-based drugs with potential clinical application.
\end{abstract}

Keywords: hydroxyapatite nanoparticles, DNAzymes, latent membrane protein 1, transfection efficiency, cellular uptake

\section{Introduction}

Epstein-Barr virus (EBV) has been closely linked with the oncogenesis of many cancers, including nasopharyngeal carcinoma. EBV-encoded latent membrane protein 1 (LMP1) is considered to be one of the key oncoproteins contributing to EBV-mediated tumorigenesis. Genetic manipulation of LMP1 expression has been suggested as a novel strategy for the treatment of EBV-associated carcinomas. ${ }^{1,2}$

DNAzymes are synthetic, single-stranded DNA catalysts that bind to their complementary sequence in a target mRNA by Watson-Crick base pairing and subsequently cleave the mRNA at predetermined phosphodiester linkages. ${ }^{3-5}$ Many studies have demonstrated that delivery of specific DNAzymes targeting tumor-associated genes exerts anticancer effects, supporting the efficiency of specific DNAzymes in downregulation of appropriate genes and highlighting DNAzymes as potential genotherapeutic molecules. ${ }^{6-12}$ In our previous studies, we discovered that EBV-LMP1-targeted DNAzymes could downregulate LMP1 expression, resulting in inhibition of cell 
proliferation, promotion of apoptosis, and enhancement of radiosensitivity both in vitro and in vivo. ${ }^{13-16}$

Active DNAzymes must penetrate the cell membrane, be appropriately trafficked in cells, enter the nucleus, and locate their target mRNA before achieving their biological function. ${ }^{17,18}$ However, due to their poor pharmacokinetic properties and biological permeability restrictions, therapeutic use of DNAzymes is dependent on the capability of delivery systems. In recent years, much effort has been made to develop highly effective, efficient, and safe vectors that provide a stable complex and protect payloads from degradation, thereby ensuring delivery of DNAzymes to target cells. ${ }^{19}$

The rise of nanotechnology has provided new methods and tools for the study of gene carriers. ${ }^{20}$ Among these, calcium phosphate nanoparticles have emerged as a potential vector for nonviral gene delivery. ${ }^{21-23}$ Hydroxyapatite $\left[\mathrm{Ca}_{10}(\mathrm{OH})_{2}\left(\mathrm{PO}_{4}\right)_{6}, \mathrm{HA}\right]$, a ceramic compound with a composition similar to that of mammalian bone and the dentin mineral compartment, has the advantages of absorbability and a high binding affinity with a variety of molecules, excellent biocompatibility, low cytotoxicity, nonimmunogenicity, lack of oncogenicity, and relatively high loading capacity and transfection efficiency. ${ }^{24,25}$ Nanohydroxyapatite particles (nHAP) incorporating $\mathrm{pEGFP-N1}$ are reportedly able to deliver DNA into gastric cancer cells without any significant cytotoxicity. ${ }^{26}$ Tan et $\mathrm{al}^{27}$ discovered that nHAP modified with protamine could improve the efficiency of gene transfection. Sun et $\mathrm{al}^{28,29}$ successfully used nHAP to deliver the NT-3 gene into the cochlear neurons of guinea pigs both in vitro and in vivo, and further demonstrated that surface modification of nHAP with polyethylenimine carrying specific genetic materials could pass through the intact round window membrane of the chinchilla with high transfection efficiency and low toxicity. Yan-Zhong et $\mathrm{al}^{30}$ used arginine-modified nanohydroxyapatite to change the surface charge of nHAP, thereby improving adsorption capacity in human epithelial cells. These studies demonstrate that nHAP may be a potentially effective and safe gene vector with possible clinical application.

In this study, we designed and prepared arginine-modified hydroxyapatite nanoparticles (Arg-nHAP) and examined the absorption efficiency of Arg-nHAP and DZ1 in vitro. We demonstrated that Arg-nHAP can efficiently deliver DNAzyme into cells, release it, and have biological functions both in vitro and in vivo. We further elucidated the mechanisms of cellular uptake and intracellular trafficking of the Arg-nHAP/DZ1 complex as an energy-dependent endocytotic process.

\section{Materials and methods Materials}

The chemicals, inhibitors, transfection reagents, and cell culture media used in these experiments were sourced as follows: fluorescein isothiocyanate (FITC)-labeled DZ1 (FITC-DZ1) and control DNAzyme (CON) were synthesized by Oligos Etc Inc (Portland, OR, USA); Lipofectamine ${ }^{\mathrm{TM}}$ 2000, ProLong ${ }^{\circledR}$ gold antifade reagent with DAPI $\left(4^{\prime}, 6-\right.$ diamidino-2-phenylindole), and trypsin-EDTA were from Invitrogen Life Technologies (Grand Island, NY, USA); high-performance liquid chromatography grade filipin III $(>85 \%)$, phenylarsine oxide $(\geq 97 \%)$, MTS (3-(4,5-dimethylthiazol-2-yl)-5-(3-carboxymethoxyphenyl)-2-(4sulfophenyl)-2H-tetrazolium), and sodium azide were from Sigma-Aldrich (St Louis, MO, USA); 2-deoxy-D-glucose was from Tokyo Chemical Industry (Tokyo, Japan); Fugene HD was from Roche (Basel, Switzerland); and fetal bovine serum was from Gibco (Grand Island, NY, USA).

\section{Design and synthesis of DZI}

DZ1 was designed to be effective in suppressing expression of the target protein LMP $1 .{ }^{13} \mathrm{CON}$ was designed based on the sequence of DZ1 by introducing two mutations in the catalytic core at positions 6 and $7\left(5^{\prime}-3^{\prime}\right)$. To determine localization of DZ1 in vitro and in vivo, DZ1 was labeled with FITC at the $5^{\prime}$ end.

\section{Preparation and characterization of Arg-nHAP}

Arg-nHAP was synthesized at the State Key Laboratory for Powder Metallurgy of Central South University by a chemical coprecipitation hydrothermal technique. First, $0.2 \mathrm{~mol} / \mathrm{L} \mathrm{Ca}$ $\left(\mathrm{NO}_{3}\right)_{2}$ and $0.2 \mathrm{~mol} / \mathrm{L}\left(\mathrm{NH}_{4}\right)_{2} \mathrm{HPO}_{4}$ solutions were mixed at a ratio of 5 to $3(\mathrm{v} / \mathrm{v}, \mathrm{Ca}$ to $\mathrm{P}$ mole ratio of 1.67) with arginine (4\%) preadded to the phosphate solution. The reaction temperature was $60^{\circ} \mathrm{C}$, and the $\mathrm{pH}$ of the mixture was adjusted to $10-11$ by ammonia water. After stirring for 30 minutes, the mixed solution was poured into a Teflon ${ }^{\circledR}$-lined stainless steel autoclave and underwent hydrothermal treatment at $170^{\circ} \mathrm{C}$ for 5 hours. After filtration and drying, crystalline Arg-nHAP was obtained. ${ }^{26,29}$

Crystalline Arg-nHAP was diluted to $5 \mathrm{mg} / \mathrm{mL}$ using ultrasonic dispersion for 60 minutes (ultrasonic homogenizer, VC500/750, Sonics \& Materials, Inc., Newtown, CT, USA) and was observed for 2 hours until it appeared layered and milky. Finally, the Arg-nHAP suspension was stored at $4^{\circ} \mathrm{C}$ after autoclaving. The particle size was measured using a 
transmission electron microscope (JEM-2100F, JEOL, Tokyo, Japan). The zeta potential was measured by a Zetasizer Nano ZS (Malvern Instrument Company, Malvern, UK). Arg-nHAP phase analysis was measured using an X-ray diffractor (D-Max/2550VB+, Rigaku, Tokyo, Japan) with $\mathrm{Cu} \mathrm{K} \alpha$ radiation $(\lambda=1.54178 \mathrm{~A}, 40 \mathrm{kV}, 30 \mathrm{~mA})$. The scanning angle/speed was $25^{\circ}-55^{\circ} / 2.4^{\circ}$ per minute and $5^{\circ}-75^{\circ} / 5^{\circ}$ per minute.

\section{Absorption efficiency of DZI and Arg-nHAP}

The absorption efficiency of Arg-nHAP and DZ1 was determined by centrifugation assay. The Arg-nHAP/DZ1 complex was prepared by mixing 100-750 $\mu \mathrm{g}$ Arg-nHAP solution with 5-60 $\mu \mathrm{g}$ DZ1 followed by incubation on a thermostat shaker $\left(500 \mathrm{rpm}, 21^{\circ} \mathrm{C}\right)$ for one hour. The mixtures were centrifuged $\left(13,000 \mathrm{~g}, 4^{\circ} \mathrm{C}\right)$ for 10 minutes. The nonadsorbed DNAzyme was quantitated in the supernatant by ultraviolet spectrophotometry at $260 \mathrm{~nm}$ using a nucleic acid analyzer (Beckman Coulter Inc, Fullerton, CA, USA).

\section{DZI release in vitro}

DZ1 release from Arg-nHAP/DZ1 was determined using a centrifugation assay. DZ1 was labeled with FITC at the $5^{\prime}$ end. The Arg-nHAP/DZ1 complex was prepared in Roswell Park Memorial Institute (RPMI) 1640 medium or in cytoplasm, plated in a 96-well Nunc plate (SigmaAldrich), and incubated at $37^{\circ} \mathrm{C}$ for $0,8,12,24$, and 48 hours, respectively. The mixtures were centrifuged $(20,000 \mathrm{~g}$, $4^{\circ} \mathrm{C}$ ) for 15 minutes. The DZ1 released was quantitated in the supernatant using a multifunctional microplate reader (Varioskan Flash, 3001, Thermo Fisher Scientific, Waltham, MA, USA) at $488 \mathrm{~nm} / 525 \mathrm{~nm}$.

\section{Cell culture}

CNE1 is a poorly differentiated EBV-negative nasopharyngeal squamous carcinoma cell line and CNE1-LMP1 is a stable LMP1-integrated nasopharyngeal carcinoma cell line. ${ }^{15}$ All cells were cultured in RPMI 1640 medium with $10 \%$ fetal bovine serum. All media were supplemented with $100 \mathrm{IU} / \mathrm{mL}$ penicillin, $100 \mu \mathrm{g} / \mathrm{mL}$ streptomycin, and $2 \mathrm{mmol} / \mathrm{L} \mathrm{L}$-glutamine. All cultures were incubated in a humidified atmosphere containing $5 \% \mathrm{CO}_{2}$ at $37^{\circ} \mathrm{C}$.

\section{Arg-nHAP/DZI transfection of CNEI-LMPI cells}

Prior to transfection, $2.0 \times 10^{5}$ CNE1-LMP1 cells per well were seeded in a six-well plate in RPMI 1640 medium with $10 \%$ fetal bovine serum and incubated at $37^{\circ} \mathrm{C}$ until
$60 \%-70 \%$ confluence at the time of transfection. The ArgnHAP/DZ1 complex ( $40 \mu \mathrm{g}$ DZ1 to $400 \mu \mathrm{g}$ Arg-nHAP) was prepared as described above. Lipofectamine 2000 or Fugene HD complexes containing the same amount of FITC-DZ1 were prepared as a comparison following the manufacturer's protocol. The complexes were then added to the cells and incubated at $37^{\circ} \mathrm{C}$ for 4 hours, followed by addition of complete medium to the wells for further experiments.

\section{FACS analysis}

The transfected CNE1-LMP1 cells were incubated at $37^{\circ} \mathrm{C}$ for 4 hours. The cells were washed with phosphate-buffered saline, harvested by treatment with trypsin-EDTA, resuspended in ice-cold phosphate-buffered saline (pH 7.4), and examined immediately using a FACSort flow cytometer (FACS, Becton Dickinson, Franklin Lakes, NJ, USA). After 20,000 total events were acquired per sample, cells were defined by assessing cell size and granularity in the forward scatter and side scatter channels. FITC fluorescence in the cell population was expressed as the geometric mean of positive events after subtraction of background fluorescence. The percentage of FITC-positive cells was determined using the CellQuest software program (Becton Dickinson).

\section{Cell viability assay}

The MTS assay was used to measure cell cytotoxicity. CNE1LMP1 cells were plated at a density of $1.0 \times 10^{4}$ cells/well in 96-well plates containing RPMI 1640 medium with $10 \%$ fetal bovine serum and incubated at $37^{\circ} \mathrm{C}$ until $60 \%-70 \%$ confluence at the time of transfection. Arg-nHAP/DZ1, Lipofectamine 2000/DZ1, or Fugene HD/DZ1 complexes were prepared as described above (final mass of DZ1, $40 \mu \mathrm{g}$ ). Twenty-four hours later, the complexes were added to the cells and incubated at $37^{\circ} \mathrm{C}$ for 24 and 48 hours, respectively. Next $20 \mu \mathrm{L}$ of MTS was added to each well and incubated at $37^{\circ} \mathrm{C}$. The absorbance of each well was read on a microplate reader at $490 \mathrm{~nm}$.

\section{Mechanism studies}

Cells were seeded in a 12-well plate at a density of $6.0 \times 10^{4}$ cells/well one day prior to transfection. Twentyfour hours after seeding, the cells were pretreated with inhibitors. The concentrations of the inhibitors were chosen based on previous studies, ${ }^{31-34} \mathrm{ie}, 10 \mathrm{mM}$ sodium azide and $50 \mathrm{mM}$ 2-deoxy-D-glucose for one hour; $0.15 \mu \mathrm{M}$ phenylarsine oxide for 10 minutes; and $1.25 \mu \mathrm{g} / \mathrm{mL}$ of filipin for one hour. The effect of low temperature on the cellular uptake of the complex was investigated at $4^{\circ} \mathrm{C}$. Following 
pretreatment, the cells were transfected with the Arg-nHAP/ FITC-DZ1 complex and analyzed by FACSort flow cytometry as described above.

\section{Confocal microscopic analysis}

Cells were plated in four-chamber polystyrene vessels on glass slides (Falcon ${ }^{\mathrm{TM}}$, BD Biosciences, Bedford, MA, USA) at a density of $4.0 \times 10^{4}$ cells per chamber one day prior to transfection. Twenty-four hours after seeding, the cells were transfected with the Arg-nHAP/DZ1 complex. After incubated at $37^{\circ} \mathrm{C}$ for 4 hours, the cells were fixed with $4 \%$ paraformaldehyde in phosphate-buffered saline at $4^{\circ} \mathrm{C}$ for 30 minutes. The cells were then washed with cold phosphate-buffered saline before their nuclei were stained with DAPI. Each slide was then sealed with a cover slip (Fisher Scientific, Pittsburgh, PA, USA). All slides were protected from light and stored at $4^{\circ} \mathrm{C}$ for further analysis using confocal microscopy (Leica TCS SP5, Nussloch, Germany).

\section{Western blot analysis}

The cells were transfected as described above and incubated for 48 hours at $37^{\circ} \mathrm{C}$, then lysed in lysis buffer. After protein quantification using the bicinchoninic acid assay (Pierce Chemicals, Rockford, IL, USA), total protein was resolved on $10 \%$ polyacrylamide sodium dodecyl sulfate gel and transferred onto a nitrocellulose membrane by electroblotting. The membrane was incubated in blocking buffer for 2 hours, followed by incubation with a primary antibody against LMP1 (Dako, Carpinteria, CA, USA) (1:250) or $\beta$-actin $(1: 1000)$ (Santa Cruz Biotechnology, San Diego, CA, USA) as an internal control overnight at $4^{\circ} \mathrm{C}$. The membrane was washed in Tris-buffered saline Tween ${ }^{\circledR} 20$ and further incubated with a secondary antibody (1:2000, anti-LMP1, Dako, M0897). Protein expression was determined using a supersignal chemiluminescence system (Pierce Chemicals) followed by exposure to autoradiographic film.

\section{Xenograft model of human nasopharyngeal carcinoma in nude mice}

Athymic BALB/C nude mice (aged 4-6 weeks, female) were injected subcutaneously with $5 \times 10^{6} \mathrm{CNE} 1-$ LMP1 cells in $0.2 \mathrm{~mL}$ of RPMI 1640 medium. Tumor volumes were determined according to the formula of length $\times$ width $\times$ height $\times(\pi / 6)$ using a digital caliper. ${ }^{35}$ When the tumor volume reached $60-110 \mathrm{~mm}^{3}$, the animals were randomly divided into five groups $(\mathrm{n}=5$ each) to receive an intratumoral injection of $20 \mu \mathrm{L}$ saline containing $100 \mu \mathrm{g}$ of
DZ1 or CON adsorbed by $3 \mu \mathrm{L}$ of Fugene HD or $20 \mu \mathrm{L}$ of Arg-nHAP, respectively, twice a week (six times in total). Tumor growth was monitored every 2 days. The mice were sacrificed at the end of the experiments, and tumor tissues were weighed and stored in $10 \%$ neutral-buffered formalin for histochemical examination.

\section{Immunohistochemistry}

Paraffin-embedded tissues were sectioned and stained for LMP1 using a streptavidin-biotin antibody coupled to peroxidase. Immunohistochemical staining of LMP1 was performed according to standard procedures. The immune complex was visualized with DAB solution and counterstained with hematoxylin (Sigma-Aldrich). Slides were scored for positivity (brown-stained cytoplasm) and negativity (blue nuclei) using a light microscope.

\section{Statistical analyses}

All statistical analyses were performed using Statistical Package for the Social Sciences version 11 software (SPSS Inc, Chicago, IL, USA). If not otherwise stated, the means of at least three independent experiments \pm standard deviation are shown. All data were calculated using the Student's $t$-test. A difference with $P<0.05$ was considered to be statistically significant, and $P<0.01$ was considered to be very significant.

\section{Results \\ Preparation and characterization of Arg-nHAP}

Crystalline Arg-nHAP was synthesized by a chemical coprecipitation hydrothermal technique, and its X-ray diffraction graph is shown in Figure 1B. The diffraction peaks of each sample are in agreement with the hexagonal nHAP in the P63 m space group (JCPDS 09-0432). This $\mathrm{X}$-ray diffraction pattern is consistent with that of standard hydroxyapatite. ${ }^{36}$ No other phases could be detected, indicating that the sample was pure nHAP crystal. The size and morphology of the nHAP crystal was identified in transmission electron micrographs (Figure 1A and C), and it could be seen that the Arg-nHAP crystal had a rod-like shape with a diameter of 20-50 nm and length of 50-200 nm. Imaging also showed that DZ1 adsorbed with Arg-nHAP had no significant effect on morphology and size. At pH 7.4, the zeta potential of Arg-nHAP, DZ1, and Arg-nHAP/DZ1 was $25.47 \pm 2.9,-16.46 \pm 2.62$, and $18.06 \pm 1.67 \mathrm{mV}$, respectively (Figure 1D). 


\section{A}
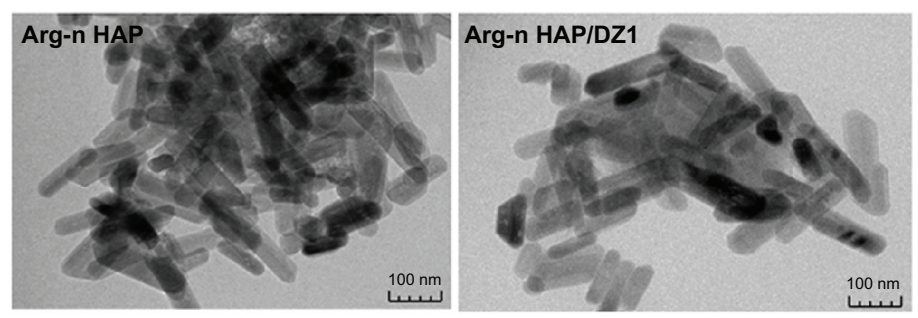

B

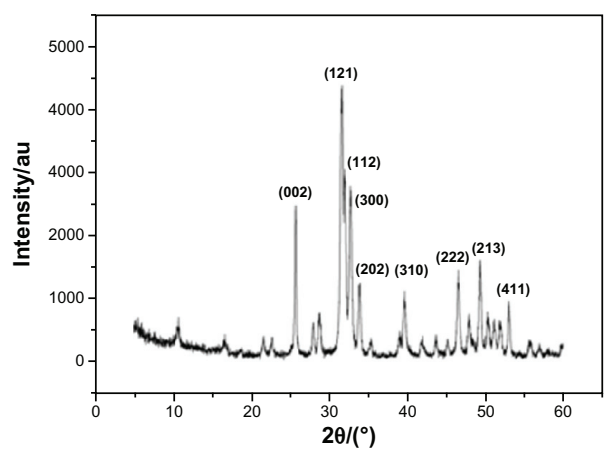

C

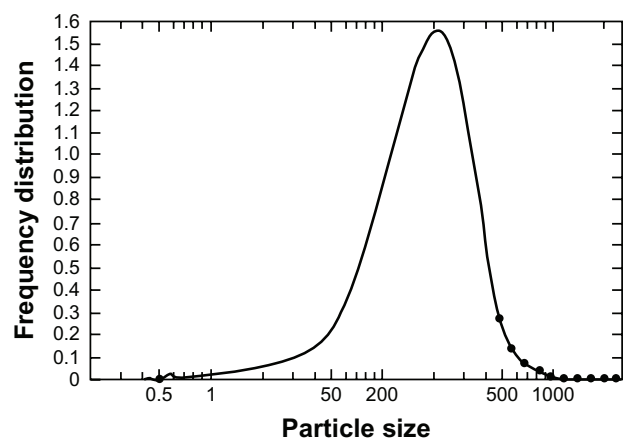

D

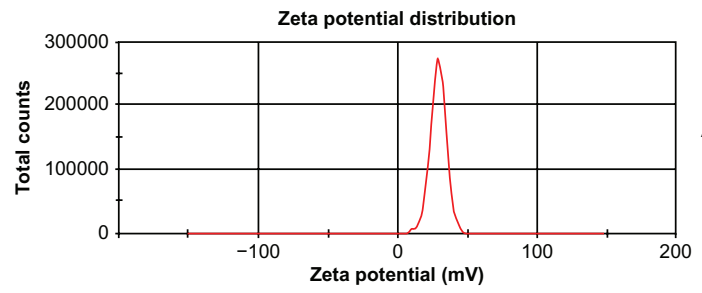

Arg-nHAP

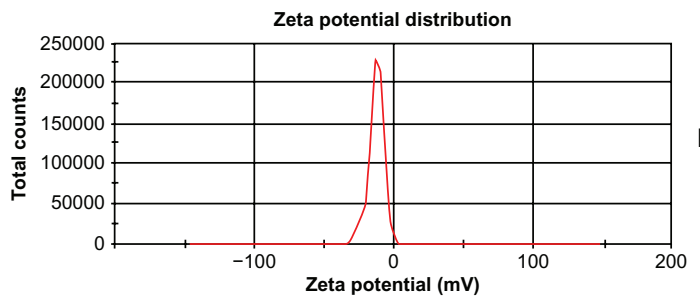

DZ1

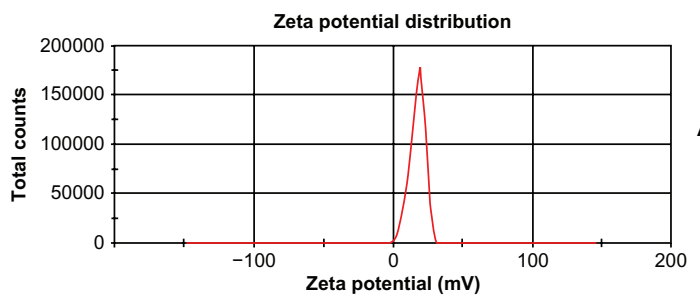

Arg-nHAP/DZ1

Figure I Characterization of Arg-nHAP and the Arg-nHAP/DZI complex ( $40 \mu \mathrm{g}$ DZI to $400 \mu \mathrm{g}$ Arg-nHAP). (A) Observation of particle size and dispersion status of Arg-nHAP using transmission electron microscopy. Scale bars represent $100 \mathrm{~nm}$. (B) Arg-nHAP phase analysis was measured by X-ray diffraction. (C) Graph of laser particle size distribution. (D) At pH 7.4, the zeta potentials of Arg-nHAP, DZI, and Arg-nHAPIDZI, as measured by a Zetasizer Nano ZS.

Abbreviations: Arg-nHAP, arginine-modified nanohydroxyapatite particles; DZI, DNAzyme I.

\section{Absorption efficiency of DZI and Arg-nHAP}

The efficiency of DZ1 adsorbed to Arg-nHAP was assessed by centrifugation assay. DZ1 was added to different concentrations of Arg-nHAP. The complex was centrifuged, and the absorbance of the supernatant was measured by ultraviolet spectrophotometry at $260 \mathrm{~nm}$. The absorption efficiency of
Arg-nHAP and DZ1 was high, reaching almost 100\% under optimal conditions, suggesting that DZ1 could be effectively adsorbed on Arg-nHAP (Table 1). Increasing Arg-nHAP was likely to increase the total capacity of DZ1 loading. As the amount of Arg-nHAP increased to $600 \mu \mathrm{g}$, the amount of DZ1 adsorbed decreased substantially, suggesting that exclusion of DZ1 occurred due to saturation of absorption. 
Table I Encapsulation efficiency of Arg-nHAP and DZI

\begin{tabular}{llllll}
\hline $\begin{array}{l}\text { Arg-nHAP } \\
(\mu \mathrm{g})\end{array}$ & \multicolumn{6}{l}{$\begin{array}{l}\text { Encapsulation efficiency (\%) at different } \\
\text { concentration of DZI }(\mu \mathrm{g})\end{array}$} \\
\cline { 2 - 6 } & $\mathbf{5}$ & $\mathbf{1 0}$ & $\mathbf{2 0}$ & $\mathbf{4 0}$ & $\mathbf{6 0}$ \\
\hline 100 & 79.80 & 50.50 & 43.75 & 24.00 & 17.15 \\
200 & 87.40 & 78.10 & 64.20 & 34.45 & 24.75 \\
400 & 92.80 & 96.20 & 90.15 & 76.48 & 45.20 \\
600 & 98.00 & 95.40 & 91.65 & 90.48 & 70.29 \\
750 & 94.80 & 96.20 & 94.80 & 75.98 & 64.92 \\
\hline
\end{tabular}

Abbreviations: Arg-nHAP, arginine-modified nanohydroxyapatite particles; DZI, DNAzyme I.

In our preliminary study, we found that cellular uptake efficiency was optimal when $40 \mu \mathrm{g}$ of DZ1 was transfected into CNE1-LMP1 cells. Therefore, we used conditions (40 $\mu \mathrm{g}$ DZ1 to $400 \mu \mathrm{g}$ Arg-nHAP) for subsequent experiments where the absorption efficiency was $76.48 \% \pm 1.38 \%$.

\section{DZI release in vitro}

To determine whether DZ1 could be released from the ArgnHAP/DZ1 complex, we performed the release assay both in medium and in cytoplasm, which was prepared from CNE1-LMP1 cells. As showed in Figure 2, DZ1 release from Arg-nHAP/DZ1 was increased in a time-dependent manner, both in RPMI 1640 medium and in cytoplasm. Interestingly, DZ1 release from the Arg-nHAP/DZ1 in cytoplasm reached $80 \%$ at 48 hours, which was significantly higher than that in RPMI 1640 (55\%). This suggests that the cellular environment could facilitate release of DZ1 from the complex.

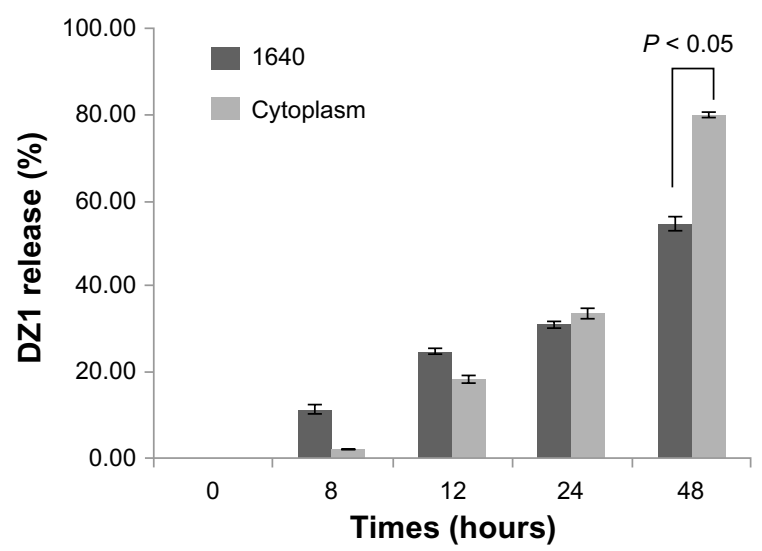

Figure 2 In vitro release profile of DZI from Arg-nHAP/DZI. The Arg-nHAP/ FITC-DZI complex was prepared in Roswell Park Memorial Institute 1640 medium or in cytoplasm, plated into a 96 -well Nunc plate, and incubated at $37^{\circ} \mathrm{C}$ for 0 , $8,12,24$, and 48 hours, respectively. The mixtures were centrifuged $(20,000 \mathrm{~g}$, $4^{\circ} \mathrm{C}$ ) for 15 minutes. The DZI released was quantitated in the supernatant using a multifunctional microplate reader at $488 \mathrm{~nm} / 525 \mathrm{~nm}$. DZI release is expressed as free fluorescein isothiocyanate-DZI in supernatant/input $D Z I \times 100$. All values are the mean of three measurements and are shown with error bars.

Abbreviations: Arg-nHAP, arginine-modified nanohydroxyapatite particles ; DZI, DNAzyme I; FITC, fluorescein isothiocyanate.

\section{Cellular uptake efficiency of Arg-nHAP/ DZI complex in CNEI-LMPI cells}

To determine cellular uptake efficiency and cellular toxicity, DZ1 was labeled with FITC at the $5^{\prime}$ end. CNE1-LMP1 cells were transfected with the complex, and the cellular uptake efficiency was analyzed by FACS. The results demonstrated that only a slight increase in fluorescence was observed in the cells treated with free FITC-DZ1. In marked contrast, approximately $74.2 \%$ of cells were FITC-positive for uptake of the Arg-nHAP/DZ1 complex. As control systems, the Fugene HD/DZ1 and Lipofectamine 2000/DZ1 complexes had a cellular uptake efficiency of $89.8 \% \pm 9.42 \%$ and $93.8 \% \pm 7.94 \%$, respectively (Figure $3 \mathrm{~A}$ ). When the transfected cells were subjected to fluorescent microscopy, no adverse effects on cell morphology were observed in cells treated with the Arg-nHAP/DZ1 complex (Figure 2B). A cytotoxicity study was carried out using MTS to quantify the survival rate of CNE1-LMP1 cells with that of control groups with the same level of DZ1. As shown in Figure 3C, the viability of Arg-nHAP was slightly better (approximately $80 \%$ ) than the commercially available transfection reagents (the cell viabilities for Fugene HD and Lipofectamine 2000 were approximately $65 \%$ and $50 \%$, respectively), suggesting that Arg-nHAP was not cytotoxic at the concentration range used in this study.

\section{Mechanisms for cellular uptake of the Arg-nHAP/DZI complex}

To determine if cellular uptake and intracellular trafficking of the Arg-nHAP/DZ1 complex was an active process or adenosine triphosphate-dependent, the transfected cells were treated with a mixture of metabolic inhibitors, ie, sodium azide and 2-deoxy-D-glucose. The effect of low temperature on cellular uptake of the complex was evaluated by incubation at $4^{\circ} \mathrm{C}$. The results show that treatment with the mixture of sodium azide and 2-deoxy-D-glucose significantly reduced the cellular uptake efficiency to $31.9 \%$ as compared with uptake in the absence of these reagents. As shown in Figure 4, a significant reduction in cellular uptake efficiency was also observed at $4{ }^{\circ} \mathrm{C}$. These results suggest that the complex was likely to be taken up by cells through an energy-dependent endocytotic pathway.

To confirm that endocytosis was relevant to cellular uptake of the Arg-nHAP/DZ1 complex, the cells were pretreated with the endocytosis inhibitors, filipin or phenylarsine oxide, prior to transfection. Phenylarsine oxide is a potent 

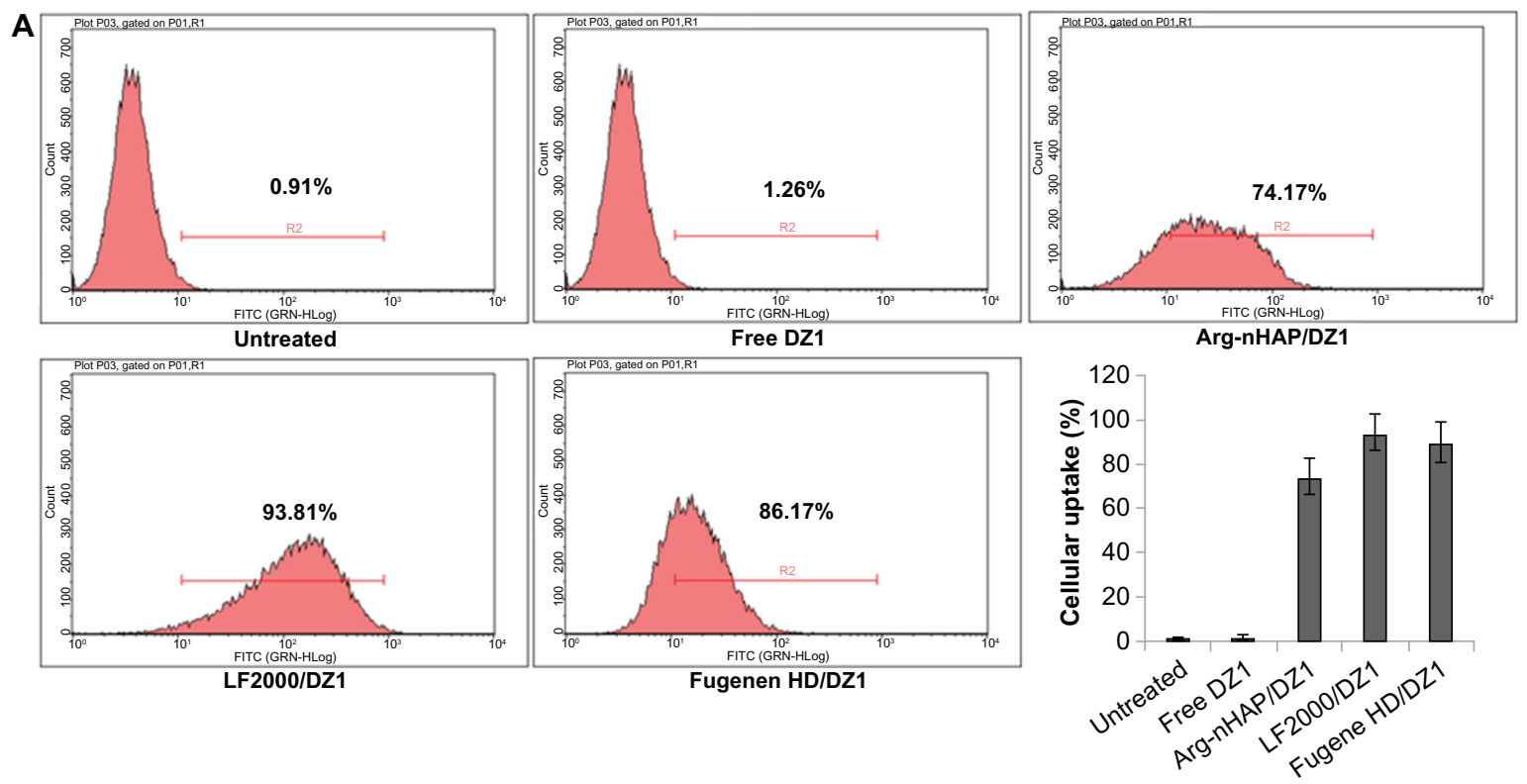

B
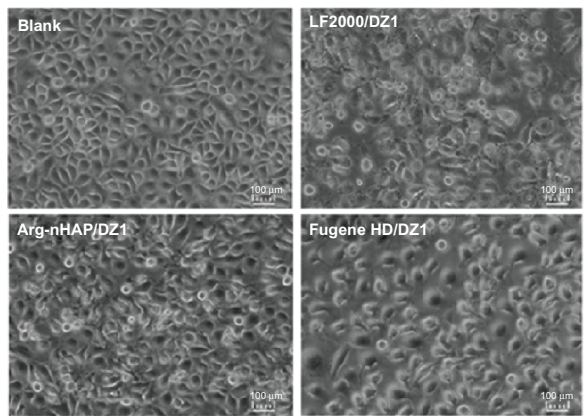

C

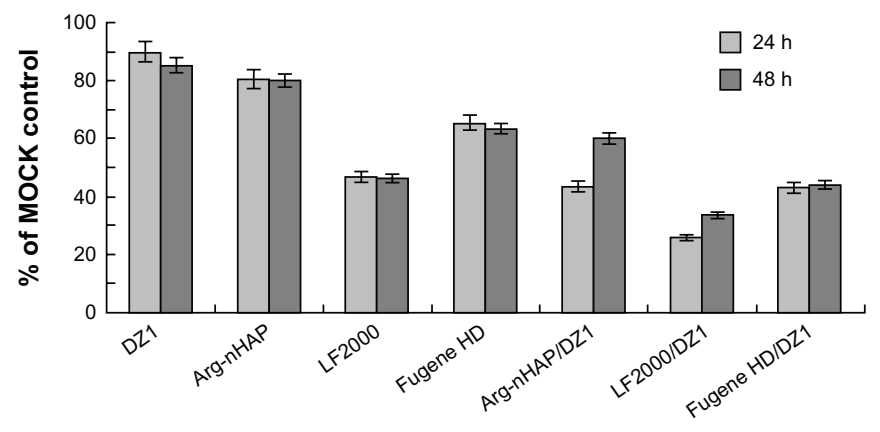

Figure 3 Cellular uptake efficiency of the Arg-nHAP/DZI complex, cell morphology, and viability. (A) Transfected cells were harvested and analyzed using FACS (DZI, FITC-labeled). FITC fluorescence in the cell population was expressed as the geometric mean of positive events after subtraction of background fluorescence (nontransfected cells). The percentage of FITC-positive cells was determined using the CellQuest software program. All values are the mean of three measurements and are shown with error bars. (B) Transfected cells were subjected to fluorescent microscopy, (200x). (C) MTS assay was used to assess cellular viability of CNEI-LMPI cells 24 and 48 hours following treatment with DZI, Arg-nHAP, Lipofectamine ${ }^{\circledR}$ 2000, Fugene, Arg-nHAP/DZI, Lipofectamine 2000/DZI and Fugene/DZI. The absorbance of each well was read on a microplate reader at $490 \mathrm{~nm}$, and the results indicating the cell viability were plotted as the percentage over controls (MOCK cells).

Abbreviations: FITC, fluorescein isothiocyanate; Arg-nHAP, arginine-modified nanohydroxyapatite particles; DZI, DNAzyme I; FACS, fluorescence activated cell sorting; MTS, 3-(4,5-dimethylthiazol-2-yl)-5-(3-carboxymethoxyphenyl)-2-(4-sulfophenyl)-2H-tetrazolium; LF2000, lipofectamine 2000.

inhibitor of clathrin-mediated endocytosis, whereas filipin inhibits the raft/caveolae pathway. Preincubation of cells with phenylarsine oxide $0.15 \mu \mathrm{M}$ resulted in significantly reduced cellular uptake of the Arg-nHAP/DZ1 complex (36.7\%). However, phenylarsine oxide did not completely inhibit active uptake of the complex when compared with cellular uptake efficiency at $4^{\circ} \mathrm{C}$ (Figure 4). Filipin $1.25 \mu \mathrm{g} / \mathrm{mL}$ also had a distinct effect on cellular uptake efficiency, with a reduction to approximately $50 \%$. Using phenylarsine oxide and filipin, we demonstrated that endocytosis of the Arg-nHAP/DZ1 complex is both clathrin-dependent and caveolae-dependent. 


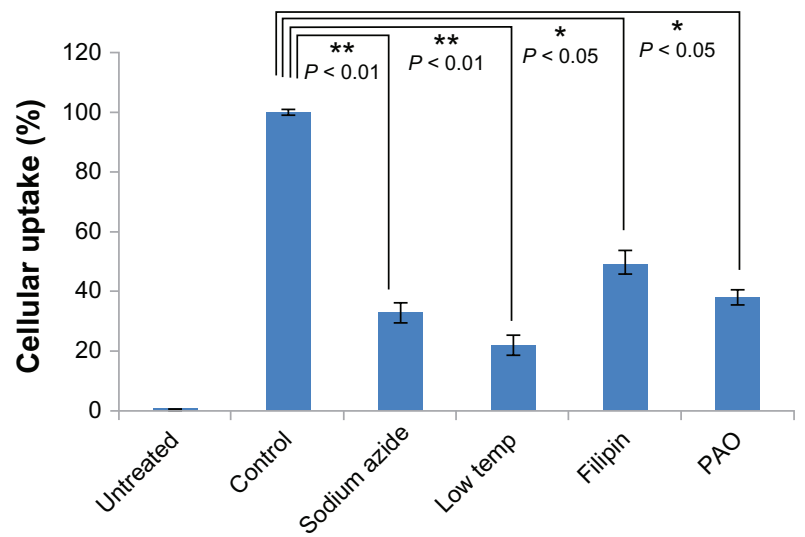

Figure 4 Cellular uptake of the Arg-nHAP/DZI complex in the presence of specific endocytic inhibitors. Cells were pretreated with inhibitors and transfected with the Arg-nHAP/FITC-DZI complex. Concentrations of the inhibitors are as follows: $20 \mathrm{mM}$ sodium azide and $50 \mathrm{mM}$ 2-deoxy-D-glucose for one hour; $0.15 \mu \mathrm{M}$ PAO for 10 minutes; and $1.25 \mu \mathrm{g} / \mathrm{mL}$ of filipin for one hour. The effect of low temperature on cellular uptake was investigated at $4^{\circ} \mathrm{C}$. All values are the mean of three measurements and are shown with error bars.

Abbreviations: FITC, fluorescein isothiocyanate; Arg-nHAP, arginine-modified nanohydroxyapatite particles; PAO, phenylarsine oxide; DZI, DNAzyme I.

\section{Intracellular localization}

\section{of the Arg-nHAP/DZI complex}

To visualize the intracellular localization of the complex in CNE1-LMP1 cells, we utilized confocal microscopy to examine the transfected cells. Consistent with the results of FACS analysis, free FITC-CON added to the culture media was poorly internalized by the cells, and confocal images revealed very limited localization in punctate cytoplasmic regions. When the cells were treated with the Arg-nHAP/FITC-DZ1 complex for 24 hours, the bright, punctate fluorescence of FITC was distributed in the cytoplasm and nuclear region, as shown in the merged picture and the FITC image (Figure 5), and the same intracellular localization was found in the Lipofectamine 2000/DZ1 and Fugene HD/DZ1 controls, suggesting successful trafficking of DZ1 to the nucleus.

\section{Downregulation of LMPI expression in CNEI-LMPI cells}

We had previously demonstrated that DZ1 transfection of LMP1-expressing cells resulted in downregulation of expression of the target gene, LMP $1 .^{13,15}$ To examine whether ArgnHAP could deliver a biologically active payload to cells, we transfected the Arg-nHAP/DZ1 complex into the cells and assayed DZ1-mediated inhibition of LMP1 expression. The data in Figure 6 show that DZ1 efficiently inhibited LMP1 expression when compared with CON-transfected and nontreated cells $(P<0.01)$, suggesting that DZ1 was successfully taken up into the cells, released into the cytoplasm, and suppressed LMP1 expression in cells.

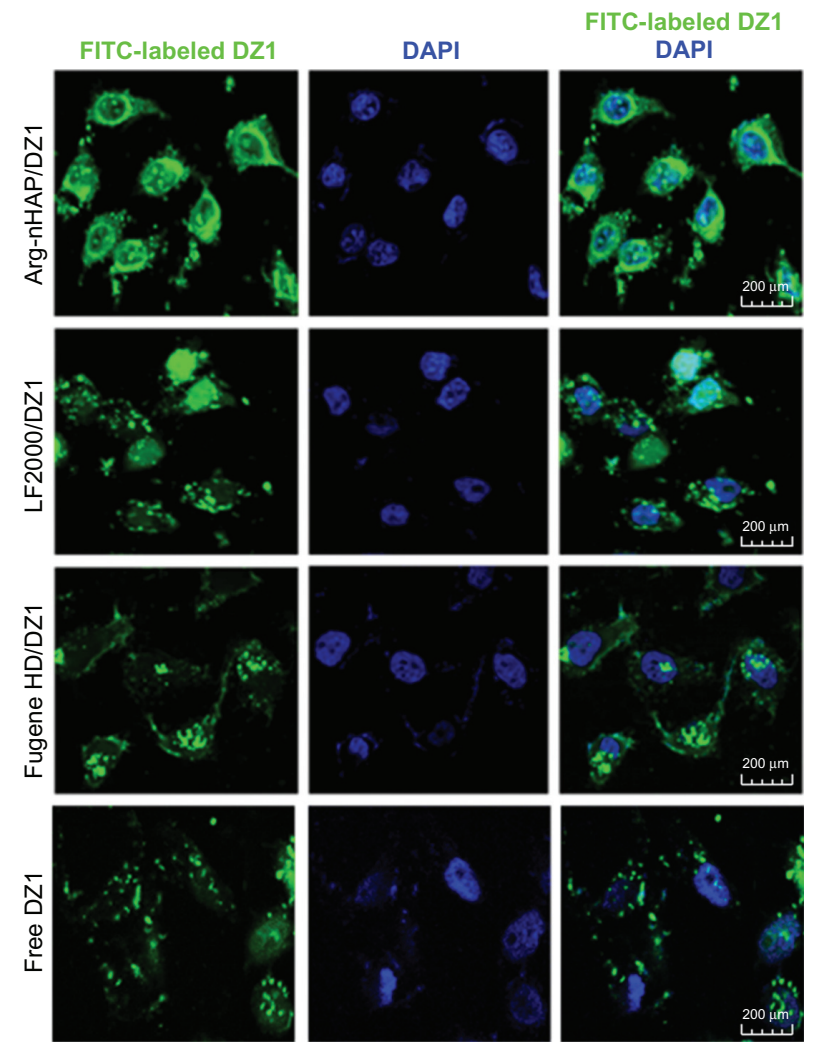

Figure 5 Intracellular localization of the Arg-nHAP/DZI complex in CNEILMPI cells. Cells were fixed with $4 \%$ paraformaldehyde at $4^{\circ} \mathrm{C}$ for 30 minutes. Nuclei were stained with DAPI (200x).

Abbreviations: Arg-nHAP, arginine-modified nanohydroxyapatite particles; DAPI, 4',6-diamidino-2-phenylindole; DZI, DNAzyme I; LF2000, lipofectamine 2000; FITC, fluorescein isothiocyanate.

\section{Antitumor effects and downregulation of LMPI expression in a CNEI-LMPI xenograft model in nude mice}

To determine whether the Arg-nHAP/DZ1 complex could downregulate LMP1 expression in vivo, we pre-established a CNE1-LMP1 xenograft model in nude mice to explore the effects of the complex on tumor growth. Fugene HD was used as the commercial control in vivo experiments due to its low cytotoxicity. As shown in Figure 7 and Table 2, Arg-nHAP/ DZ1 significantly inhibited tumor growth compared with ArgnHAP/CON and saline $(P<0.05)$. In addition, Fugene HD/ DZ1 and CON had only a marginal effect on tumor growth $(P>0.5)$. These data suggest that Arg-nHAP may be used as a delivery vehicle for nucleic acid-based agents.

To demonstrate that LMP1 expression was indeed inhibited in the tumor tissues, immunohistochemical staining was done on cross-sections of paraffin-embedded formalin-fixed tissues from the different treatment groups. The results show that LMP1 expression in tumor tissues treated with the ArgnHAP/DZ1 complex was significantly reduced compared with CON (Figure 8), indicating that Arg-nHAP was delivered 

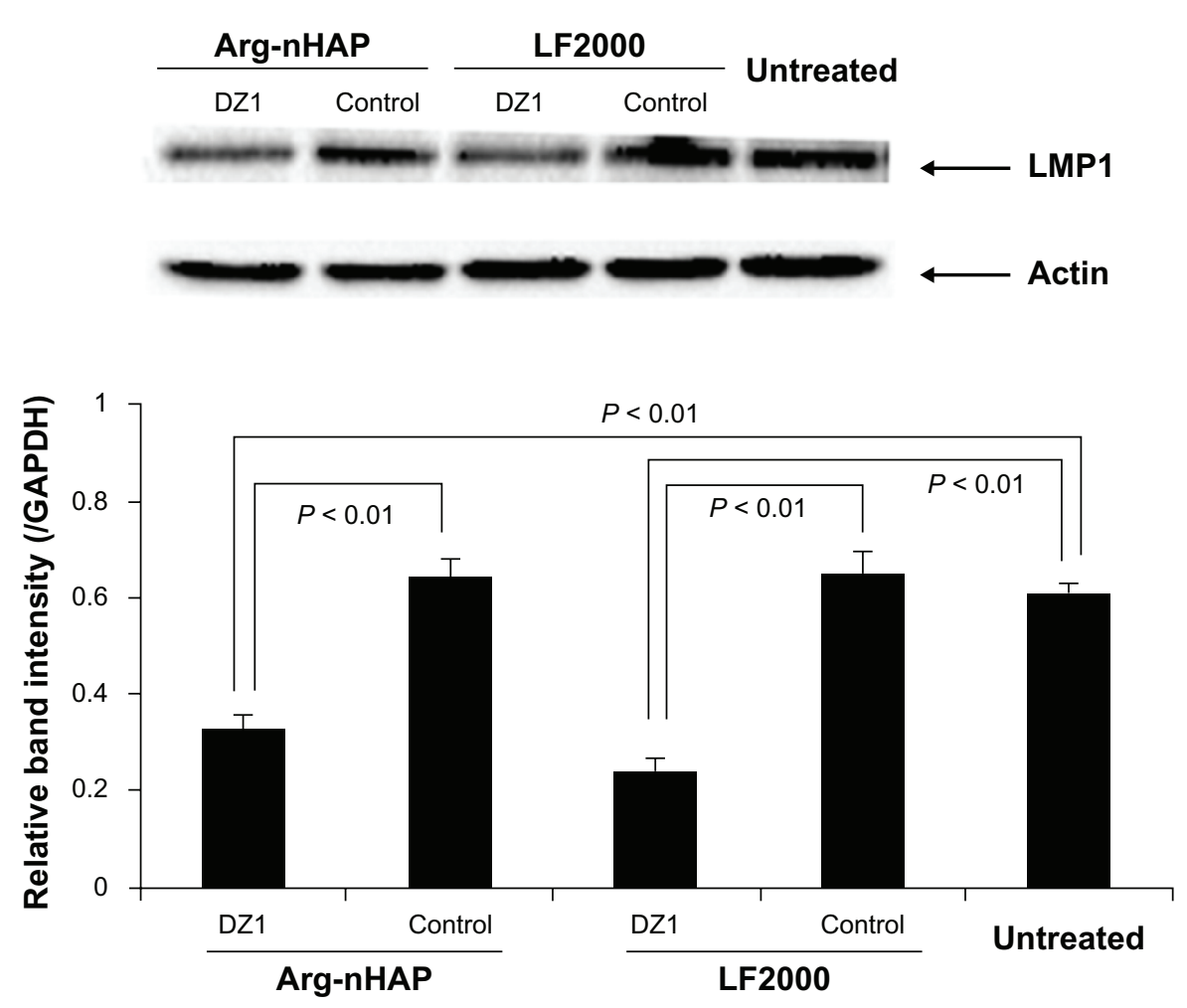

Figure 6 Inhibition of LMPI protein expression in CNEI-LMPI cells using DZI. Cells were transfected with DZI or control (40 $\mu \mathrm{g})$. All values are the mean of three measurements and are shown with error bars.

Abbreviations: Arg-nHAP, arginine-modified nanohydroxyapatite particles; GAPDH, glyceraldehyde 3-phosphate dehydrogenase; LMPI, latent membrane protein I; DZI, DNAzyme I; LF2000, lipofectamine 2000.

successfully to the tumor cells and that DZ1 could locate its target mRNA and exert its biological effects in vivo.

\section{Discussion}

The characteristics of DNAzymes, including their remarkable catalytic efficiency and specificity, make them an attractive approach to gene therapy. One of the problems for potential

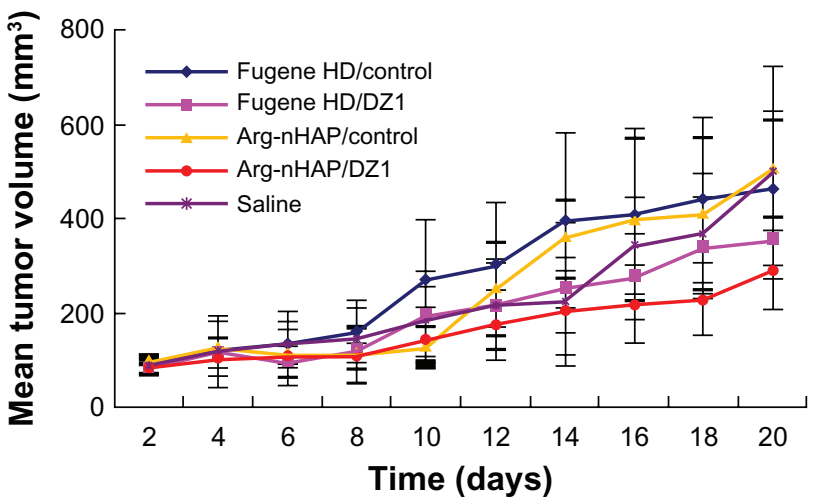

Figure 7 Tumor growth curve of each group in a nasopharyngeal carcinoma xenograft model. When the tumor volume reached $60-110 \mathrm{~mm}^{3}$, the animals were injected intratumorally with $100 \mu \mathrm{g}$ of DZI or CON adsorbed by $3 \mu \mathrm{L}$ of Fugene HD or $20 \mu \mathrm{L}$ of Arg-nHAP ( $20 \mu \mathrm{g}$ per injection) twice a week (six times total). The tumor volume was measured twice a week. Tumor tissues were harvested and weighed at the end of the experiment.

Abbreviations: Arg-nHAP, arginine-modified nanohydroxyapatite particles; CON, control DNAzyme; DZI, DNAzyme I. therapeutic use of DNAzymes is the lack of efficient delivery systems. Such systems should provide efficient delivery of DNAzymes into target cells, with protection from nucleases, promotion of endosomal release of DNAzymes into the cytoplasm, and location of the target mRNA. ${ }^{37}$ Commercially available liposomes have demonstrated high cellular uptake efficiency and capacity in vitro tests..$^{38}$ However, in vivo applications, they are usually accompanied by unwanted regional and systemic toxicity. ${ }^{39}$

Calcium phosphate nanoparticles represent a unique class of nonviral vectors that have attracted increasing attention as new candidates for nonviral vectors in gene therapy. ${ }^{40-43}$

Table 2 Statistical analysis of mean tumor volume

\begin{tabular}{lllll}
\hline Group & Cases & Mean \pm SD & P value & \\
\hline Fugene HD/control & 5 & $464.74 \pm 164.78$ & $0.730^{\mathrm{a}}$ & $0.570^{\mathrm{e}}$ \\
Fugene HD/DZI & 5 & $354.83 \pm 107.58$ & $0.309^{\mathrm{b}}$ & \\
Arg-nHAP/control & 5 & $505.82 \pm 102.82$ & $0.940^{\mathrm{c}}$ & $0.024^{\mathrm{f}, *}$ \\
Arg-nHAP/DZI & 5 & $290.86 \pm 85.40$ & $0.037^{\mathrm{d}, *}$ & \\
Saline & 5 & $498.83 \pm 188.82$ & & \\
\hline
\end{tabular}

Notes: aFugene HD/control compared with saline; bfugene HD/DZI compared with

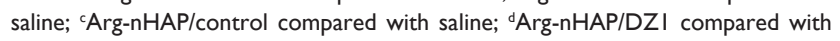
saline; 'fugene HD/control compared with Fugene HD/DZI; ${ }^{\mathrm{f}} \mathrm{Arg}-\mathrm{nHAP} / \mathrm{control}$ compared with Arg-nHAP/DZI. *Statistically significant $(P<0.05)$ versus control. Abbreviations: Arg-nHAP, arginine-modified nanohydroxyapatite particles; SD, standard deviation; DZI, DNAzyme I. 

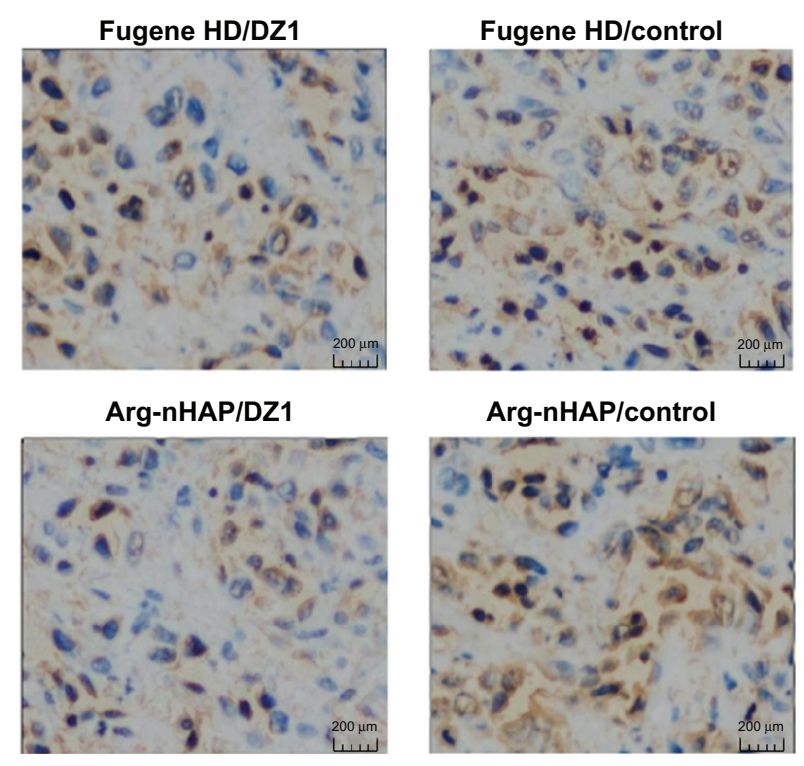

Figure 8 Immunohistochemical analyses of tumor tissues. Paraffin-embedded tissues were sectioned and stained for LMPI using a streptavidin-biotin technique coupled to peroxidase. The analysis reveals positive cytoplasmic staining for LMPI. The microphotographs represent the average number of LMPI-positive cells per $200 \times$ high-power fields for each group.

Abbreviations: Arg-nHAP, arginine-modified nanohydroxyapatite particles; DZI, DNAzyme I; LMPI, latent membrane protein I.

In the present study, we established an effective Arg-nHAP/ DNAzyme delivery system in which nHAP was modified with arginine conjugated to a guanidyl group $\left(-\left(\mathrm{CH}_{2}\right)_{3}\right.$ $\left.\mathrm{NHC}\left(\mathrm{NH}_{2}\right)^{+}\right)$on the surface of Arg-nHAP to prevent crystal growth. This system was shown to facilitate absorption of DNAzyme effectively, with cellular uptake of covalently attached particles to deliver active DNAzyme into cells and animals. The observed biological activity both in vitro and in vivo indicates that DZ1 delivered by nHAP should have been released from the complex in the cytoplasm and located its target mRNA to exert its biological effects.

In comparison with Lipofectamine 2000 and Fugene HD, which have been used widely in vitro settings, Arg-nHAP showed similar cellular uptake efficiency both in test tubes and in cell cultures. However, Arg-nHAP/DNAzyme showed much more efficient delivery in vivo than did Fugene, as evidenced by the strong biological activity of Arg-nHAP/ DZ1 in suppressing nasopharyngeal tumor growth (Figure 7). This discrepancy between the in vitro and in vivo activity of Arg-nHAP and that of the commercially available transfection reagents may be due to the unique properties of the Arg-nHAP nanoparticles, including loading capacity, low toxicity, and biocompatibility. ${ }^{25,36}$

In this study, we explored the mechanisms of cellular uptake and intracellular trafficking of the Arg-nHAP/DZ1 complex. By inhibition studies using specific metabolic inhibitors, ie, sodium azide and 2-deoxy-D-glucose, which block production of adenosine triphosphate by interfering with glycolytic and oxidative metabolic pathways in cells, the complex was determined to be probably taken up by cells through an active process. ${ }^{44,45}$ Further, a significant reduction in transfection efficiency of the complex was observed at $4^{\circ} \mathrm{C}$, confirming that cellular uptake of the complex relied on an energy-dependent pathway.

Endocytosis is known to be a prevalent and major process for cellular uptake of nanomaterials. ${ }^{46,47}$ Studies have suggested the involvement of both clathrin-mediated and caveolae-mediated pathways in lipid-based and polymerbased gene delivery systems. ${ }^{48,49}$ For calcium phosphate-based gene delivery systems, both clathrin-dependent and caveolaedependent endocytosis was suggested to be involved..$^{50}$ To examine if endocytosis was relevant to cellular uptake of the Arg-nHAP/DZ1 complex, the cells were pretreated with endocytosis inhibitors, such as filipin or phenylarsine oxide, prior to transfection. Phenylarsine oxide inhibits clathrinmediated endocytosis by reacting with vicinal sulfhydryls to form stable ring structures, whereas filipin interacts with 3-b-hydroxysterols in the plasma membrane to form filipinsterol complexes and subsequently induces the filamentous caveolin-1 coat to disassemble rapidly, leading to inhibition of caveolae-mediated endocytosis. We demonstrated that treatment of cells with these inhibitors significantly reduced cellular uptake of Arg-nHAP/DZ1, suggesting that endocytosis of the Arg-nHAP/DZ1 complex was clathrin-dependent and caveolae-dependent.

The features of Arg-nHAP indicate that it may be an efficient vector for delivery of biologically active molecules. Further studies are needed to address issues such as the stability, pharmacokinetics, and toxicity of these nanoparticles before realization of their clinical potential.

\section{Conclusion}

Data from this study indicate that DZ1 was successfully encapsulated with Arg-nHAP and transfected into cells, where it was released, entered the nucleus, located the target mRNA, and inhibited LMP1 protein expression in vitro and in vivo. Arg-nHAP nanoparticles represent a promising nonviral carrier for gene therapy.

\section{Acknowledgment}

This work was supported by the Key Projects in the National Science and Technology Pillar Program during the Twelfth Five-year Plan Period (2013BAI01B07) and the National Nature Science Foundation of China $(81172188 ; 81072220$; 91129709; and 51102285). 


\section{Disclosure}

The authors report no conflicts of interest in this work.

\section{References}

1. Chou J, Lin YC, Kim J, et al. Nasopharyngeal carcinoma - review of the molecular mechanisms of tumorigenesis. Head Neck. 2008;30(7): 946-963.

2. Morris MA, Dawson CW, Young LS. Role of the Epstein-Barr virusencoded latent membrane protein-1, LMP1, in the pathogenesis of nasopharyngeal carcinoma. Future Oncol. 2009;5(6):811-825.

3. Cairns MJ, Saravolac EG, Sun LQ. Catalytic DNA: a novel tool for gene suppression. Curr Drug Targets. 2002;3(3):269-279.

4. Baum DA, Silverman SK. Deoxyribozymes: useful DNA catalysts in vitro and in vivo. Cell Mol Life Sci. 2008;65(14):2156-2174.

5. Sun LQ, Cairns MJ, Saravolac EG, Baker A, Gerlach WL. Catalytic nucleic acids: from lab to applications. Pharmacol Rev. 2000;52(3): 325-347.

6. Sun LQ, Cairns MJ, Gerlach WL, Witherington C, Wang L, King A. Suppression of smooth muscle cell proliferation by a c-myc RNAcleaving deoxyribozyme. J Biol Chem. 1999;274(24):17236-17241.

7. Lowe HC, Chesterman CN, Khachigian LM. Catalytic antisense DNA molecules targeting Egr-1 inhibit neointima formation following permanent ligation of rat common carotid arteries. Thromb Haemost. 2002;87(1):134-140.

8. Zhang L, Gasper WJ, Stass SA, Ioffe OB, Davis MA, Mixson AJ. Angiogenic inhibition mediated by a DNAzyme that targets vascular endothelial growth factor receptor 2. Cancer Res. 2002;62(19): 5463-5469.

9. Arteaga CL, Baselga J. Clinical trial design and end points for epidermal growth factor receptor-targeted therapies: implications for drug development and practice. Clin Cancer Res. 2003;9(5):1579-1589.

10. Wu Y, Yu L, McMahon R, Rossi JJ, Forman SJ, Snyder DS. Inhibition of bcr-abl oncogene expression by novel deoxyribozymes (DNAzymes). Hum Gene Ther. 1999;10(17):2847-2857.

11. Mitchell A, Dass CR, Sun LQ, Khachigian LM. Inhibition of human breast carcinoma proliferation, migration, chemoinvasion and solid tumour growth by DNAzymes targeting the zinc finger transcription factor EGR-1. Nucleic Acids Res. 2004;32(10):3065-3069.

12. Zhang G, Dass CR, Sumithran E, Di Girolamo N, Sun LQ, Khachigian LM. Effect of deoxyribozymes targeting c-Jun on solid tumor growth and angiogenesis in rodents. J Natl Cancer Inst. 2004;96(9):683-696.

13. Lu ZX, Ye M, Yan GR, et al. Effect of EBV LMP1 targeted DNAzymes on cell proliferation and apoptosis. Cancer Gene Ther. 2005;12(7): 647-654

14. Liu YP, Tan YN, Wang ZL, et al. Phosphorylation and nuclear translocation of STAT3 regulated by the Epstein-Barr virus latent membrane protein 1 in nasopharyngeal carcinoma. Int J Mol Med. 2008;21(2):153-162.

15. Lu ZX, Ma XQ, Yang LF, et al. DNAzymes targeted to EBV-encoded latent membrane protein-1 induce apoptosis and enhance radiosensitivity in nasopharyngeal carcinoma. Cancer Lett. 2008;265(2):226-238.

16. Yang L, Lu Z, Ma X, Cao Y, Sun LQ. A therapeutic approach to nasopharyngeal carcinomas by DNAzymes targeting EBV LMP-1 gene. Molecules. 2010;15(9):6127-6139.

17. Dass CR, Saravolac EG, Li Y, Sun LQ. Cellular uptake, distribution, and stability of 10-23 deoxyribozymes. Antisense Nucleic Acid Drug Dev. 2002;12(5):289-299.

18. Tan ML, Choong PF, Dass CR. DNAzyme delivery systems: getting past first base. Expert Opin Drug Deliv. 2009;6(2):127-138.

19. Luo D, Saltzman WM. Synthetic DNA delivery systems. Nat Biotechnol. 2000;18(1):33-37.

20. Ditto AJ, Shah PN, Yun YH. Non-viral gene delivery using nanoparticles. Expert Opin Drug Deliv. 2009;6:1149-1160.

21. Roy I, Mitra S, Maitra A, Mozumdar S. Calcium phosphate nanoparticles as novel non-viral vectors for targeted gene delivery. Int J Pharm. $2003 ; 250(1): 25-33$.
22. Bisht S, Bhakta G, Mitra S, Maitra A. pDNA loaded calcium phosphate nanoparticles: highly efficient non-viral vector for gene delivery. Int $J$ Pharm. 2005;288(1):157-168.

23. Olton D, Li J, Wilson ME, et al. Nanostructured calcium phosphates (NanoCaPs) for non-viral gene delivery: influence of the synthesis parameters on transfection efficiency. Biomaterials. 2007;28(6): 1267-1279.

24. Loo SC, Moore T, Banik B, Alexis F. Biomedical applications of hydroxyapatite nanoparticles. Curr Pharm Biotechnol. 2010;11(4): 333-342.

25. Chen L, Mccrate JM, Lee J, Li H. The role of surface charge on the uptake and biocompatibility of hydroxyapatite nanoparticles with osteoblast cells. Nanotechnology. 2011;22(10):105708.

26. Zhu SH, Huang BY, Zhou KC, et al. Hydroxyapatite nanoparticles as a novel gene carrier. J Nanopart Res. 2004;6(2):307-311.

27. Tan K, Cheang P, Ho IA, Lam PY, Hui KM. Nanosized bioceramic particles could function as efficient gene delivery vehicles with target specificity for the spleen. Gene Ther. 2007;14(10):828-835.

28. Sun H, Jiang M, Zhu SH. In vitro and in vivo studies on hydroxyapatite nanoparticles as a novel vector for inner ear gene therapy. Zhonghua Er Bi Yan Hou Tou Jing Wai Ke Za Zhi. 2008;43(1):51-57. Chinese.

29. Wu X, Ding D, Jiang H, et al. Transfection using hydroxyapatite nanoparticles in the inner ear via an intact round window membrane in chinchilla. J Nanopart Res. 2012;14(1):1-13.

30. Yan-Zhong Z, Yan-Yan H, Jun Z, Shai-Hong Z, Zhi-You L, Ke-Chao Z. Characteristics of functionalized nano-hydroxyapatite and internalization by human epithelial cell. Nanoscale Res Lett. 2011;6:600.

31. Perumal OP, Inapagolla R, Kannan S, Kannan RM. The effect of surface functionality on cellular trafficking of dendrimers. Biomaterials. 2008;29(24-25):3469-3476.

32. Schnitzer JE, Oh P, Pinney E, Allard J. Filipin-sensitive caveolaemediated transport in endothelium: reduced transcytosis, scavenger endocytosis, and capillary permeability of select macromolecules. J Cell Biol. 1994;127(5):1217-1232.

33. Panyam J, Zhou WZ, Prabha S, Sahoo SK, Labhasetwar V. Rapid endo-lysosomal escape of poly(DL-lactide-co-glycolide) nanoparticles: implications for drug and gene delivery. FASEB J. 2002;16(10): 1217-1226.

34. Huang M, Ma Z, Khor E, Lim LY. Uptake of FITC-chitosan nanoparticles by A549 cells. Pharm Res. 2002;19(10):1488-1494.

35. Yang L, Xiao L, Ma X, et al. Effect of DNAzymes targeting Akt1 on cell proliferation and apoptosis in nasopharyngeal carcinoma. Cancer Biol Ther. 2009;8(4):366-371.

36. Balasundaram G, Sato M, Webster TJ. Using hydroxyapatite nanoparticles and decreased crystallinity to promote osteoblast adhesion similar to functionalizing with RGD. Biomaterials. 2006;27(14):2798-2805.

37. Shegokar R, A1 SL, Mishra PR. SiRNA delivery: challenges and role of carrier systems. Pharmazie. 2011;66(5):313-318.

38. Kulkarni M, Greiser U, O’Brien T, Pandit A. Liposomal gene delivery mediated by tissue-engineered scaffolds. Trends Biotechnol. 2010;28(1):28-36.

39. Masotti A, Mossa G, Cametti C, et al. Comparison of different commercially available cationic liposome-DNA lipoplexes: parameters influencing toxicity and transfection efficiency. Colloids Surf B Biointerfaces. 2009;68(2):136-144.

40. Zhu S, Zhou K, Huang B, et al. Hydroxyapatite nanoparticles: a novel material of gene carrier. Sheng Wu Yi Xue Gong Cheng Xue Za Zhi. 2005;22(5):980-984. Chinese.

41. Do TN, Lee WH, Loo CY, Zavgorodniy AV, Rohanizadeh R. Hydroxyapatite nanoparticles as vectors for gene delivery. Ther Deliv. 2012;3(5):623-632.

42. Figueiredo M, Esenaliev R. PLGA nanoparticles for ultrasound-mediated gene delivery to solid tumors. J Drug Deliv. 2012;2012:767839.

43. Loo SC, Moore T, Banik B, Alexis F. Biomedical applications of hydroxyapatite nanoparticles. Curr Pharm Biotechnol. 2010;11(4): 333-342. 
44. Zelphati O, Szoka FJ. Intracellular distribution and mechanism of delivery of oligonucleotides mediated by cationic lipids. Pharm Res. 1996;13(9):1367-1372.

45. Kakizawa Y, Furukawa S, Kataoka K. Block copolymer-coated calcium phosphate nanoparticles sensing intracellular environment for oligodeoxynucleotide and siRNA delivery. $J$ Control Release. 2004;97(2):345-356.

46. Khalil IA, Kogure K, Akita H, Harashima H. Uptake pathways and subsequent intracellular trafficking in nonviral gene delivery. Pharmacol Rev. 2006;58(1):32-45.

47. Nie H, Wang CH. Fabrication and characterization of PLGA/HAp composite scaffolds for delivery of BMP-2 plasmid DNA. J Control Release. 2007;120(1-2):111-121.
48. Rejman J, Bragonzi A, Conese M. Role of clathrin- and caveolaemediated endocytosis in gene transfer mediated by lipo- and polyplexes. Mol Ther. 2005;12(3):468-474.

49. Huth US, Schubert R, Peschka-Suss R. Investigating the uptake and intracellular fate of $\mathrm{pH}$-sensitive liposomes by flow cytometry and spectral bio-imaging. J Control Release. 2006;110(3):490-504.

50. Olton DY, Close JM, Sfeir CS, Kumta PN. Intracellular trafficking pathways involved in the gene transfer of nano-structured calcium phosphate-DNA particles. Biomaterials. 2011;32(30):7662-7670.

\section{Publish your work in this journal}

The International Journal of Nanomedicine is an international, peerreviewed journal focusing on the application of nanotechnology in diagnostics, therapeutics, and drug delivery systems throughout the biomedical field. This journal is indexed on PubMed Central, MedLine, CAS, SciSearch $\AA$, Current Contents ${ }^{\circledR} /$ Clinical Medicine,
Journal Citation Reports/Science Edition, EMBase, Scopus and the Elsevier Bibliographic databases. The manuscript management system is completely online and includes a very quick and fair peer-review system, which is all easy to use. Visit http://www.dovepress.com/ testimonials.php to read real quotes from published authors. 